\title{
Reflexiones sobre feminismos jurídicos, derecho y universidad: el caso de la formación universitaria de grado en Derecho
}

Reflections on legal feminism, rigths and university: the case of the college formation in law school

\author{
Melanie Denisse Salcedo melaniesalcedo@gmail.com \\ http://orcid.org/0000-0001-6781-6520 \\ Universidad Nacional de La Matanza/ \\ Universidad de Morón (Argentina)
}

\section{Resumen}

El presente artículo reflexiona sobre el lugar que ocupa la perspectiva de género en la formación de grado de las y los estudiantes de la carrera de Abogacía, adoptando como eje de análisis los aportes realizados por los feminismos jurídicos en tensión con la supuesta neutralidad del discurso jurídico. La ausencia de una perspectiva de género que atraviese de manera transversal la formación de grado de las abogadas y los abogados se constituye como punto de partida para dar cuenta de la emergencia pedagógica que atraviesa al Derecho, pasando revista sobre las implicancias entre género, patriarcado y discurso jurídico como aparato discursivo que normaliza y legitima posiciones dominantes en un mundo gobernado por la lógica de la dominación masculina y que la enseñanza universitaria no logra cuestionar. 
Palabras clave: Feminismos jurídicos; Derecho; discurso jurídico; enseñanza del derecho; patriarcado.

\section{Abstract}

This article reflects about the position that perspective of gender takes in the college degree of advocacy, adopting the feminist jurisprudence's contribution as center of analysis in tension with the supposed neutrality of the juridical speech. The lack of a gender's perspective that crosses the training to become a lawyer in a transversal way brings awareness of the pedagogical emergency that advocacy faces, reviewing the implications between gender, patriarchy and legal discourse, in an atmosphere of male domination where the university is not able to find the way to question.

Keywords: Feminisms jurisprudence; law; legal discourse; legal education; patriarchy.

\section{Feminismo y universidad}

El presente trabajo parte de una investigación realizada para la Carrera de Formación Docente Superior dictada por la Facultad de Filosofía y Ciencias de la Educación de la Universidad de Morón, donde se analizaron los programas de estudio de todas las asignaturas (y sus diferentes cátedras) para indagar el lugar que ocupa -de existir- la perspectiva de género en la formación de grado de las y los estudiantes de Abogacía.

De los señalamientos que realizan Bernstein y Díaz (2017: 5) y en tensión con lo que señala MacKinnon (2005) en cuanto a la pertinencia espacial de la universidad como centro de cuestionamientos, la pregunta central que emerge como síntesis de las aproximaciones preliminares que se realizan en este trabajo se encaminan a cuestionar ¿Por qué los feminismos jurídicos no entran en el "círculo reservado del conocimiento"? (Focault, 1971).

Los feminismos jurídicos realizan diversas contribuciones en torno a la neutralidad del discurso jurídico y la abstracción del sujeto de derecho para pensar la tradicional ausencia del género en la enseñanza del Derecho en Latinoamérica, remarcando cómo se producen y reproducen ciertos mecanismos para la negación de las necesidades y experiencias femeninas (1). 
Teniendo en cuenta el carácter performativo del Derecho, cuya capacidad reconoce o invisibiliza, incluye o excluye y nombra y delimita lo legal y lo ilegal, la universidad como espacio de construcción y legitimación del Derecho se erige como una pieza clave en el mapa político social (Costa, 2016; 11).

En esta línea, entendemos que las abogadas feministas latinoamericanas debemos hacer visible la emergencia pedagógica en las facultades de Derecho, en miras de lograr la inclusión de perspectivas que desafíen las nociones de sentido común que reproducen las desigualdades de género y de ese modo fomentar el pensamiento crítico de las y los estudiantes.

Coincidimos con Facio y Fries (2005: 260) en cuanto a la necesidad de repensar el Derecho para hacer de éste un instrumento que (regulando) transforme la convivencia en sociedad y desplace los modelos sexuales, sociales, económicos y políticos hacia una convivencia social basada en la aceptación de las diferencias en respeto de la diversidad.

\section{Crítica a la representación de una humanidad universal}

El movimiento feminista emerge como una organización de mujeres con una ideología plural, diversa e inacabada cuyo objetivo primero es la transformación de la situación de subordinación y opresión de la mujer. Este movimiento social e internacionalista, político e ideológico, parte de la premisa -tan simple y sorprendentemente necesaria- que las mujeres somos personas.

Los feminismos jurídicos (2) constituyen un área específica de investigación dentro del movimiento feminista surgida a partir de los años setenta en los Estados Unidos, cuya mención en plural permite dar cuenta de la variedad de posturas y propuestas que se congregan en este movimiento. Tal como señala Costa (2016) la falacia de la igualdad universal como principio jurídico, resulta un punto de partida para ingresar a la cosmovisión feminista del Derecho.

Todo universal -en este caso jurídico- implica la negación de ciertas realidades que no se corresponden con la figura abstracta creada por el Derecho. En el caso de la subordinación de las mujeres, los feminismos jurídicos señalan una serie de antecedentes históricos que ejemplifican cómo actúan los universales y el sujeto abstracto de Derecho, dando cuenta de la no correspondencia y proporcionalidad entre la igualdad formal -aquella que se le reconoce a todas las personas a través de fuentes legislativas-; la igualdad material -determinada por las circunstancias relativas al sexo, género, etnia, religión, entre otras que pese al reconocimiento 
formal impiden el goce de los derechos efectivamente reconocidos- y la igualdad estructural que se relaciona con la falta histórica de acceso a diversas instituciones de colectivos vulnerables y grupos discriminados-.

La Declaración de los Derechos de la Mujer y de la Ciudadana de 1971, redactada por la feminista francesa Olympe de Gouges, a quien ello le costara la vida, pone de manifiesto cómo el instrumento emblema de la noción de igualdad moderna -asociado a la Revolución Francesa y conocido como "La Declaración de los Derechos del Hombre y el Ciudadano" de 1789constituye una falacia que se limitaba solo a un conjunto de personas, en donde no había lugar para la mujer: los derechos reconocidos eran dignos de algunos varones, los considerados ciudadanos: blancos, adultos y propietarios.

A nivel nacional, otro ejemplo de cómo los universales del Derecho no siempre comprenden a todos en su especie, lo encontramos en la famosa Ley "Sáenz Peña" de Sufragio universal, secreto y obligatorio, en donde se evidencia cómo lo universal es una construcción social que nada tiene relación con toda la humanidad ya que, si bien esta ley es de 1912, las mujeres recién pudieron hacer uso de ese derecho en 1947 gracias a las luchas de los movimientos feministas sufragistas.

De esta manera y siguiendo a Costa (2016: 30) -quien retoma los señalamientos de Maffia (2012)- el discurso del Derecho se edifica sobre una figura central denominada "hombre", con presunciones de representación total de la humanidad, por lo que en el imaginario social el hombre -varón masculino- representa lo universal y neutral, mientras que las mujeres, como contracara representan "lo otro" del hombre, lo particular de lo universal. De allí que Derecho sea calificado por los feminismos jurídicos como discurso androcéntrico (haciendo referencia al privilegio y al dominio del varón adulto, propietario, capaz y educado por sobre otros grupos). Hacer visible esta caracterización en la formación universitaria supone permitirles a las y los estudiantes sospechar de aquello que se inscribe en una naturaleza biológica que es en sí misma una construcción social naturalizada (Bourdieu, 2000: 37).

Sostenemos que una educación superior en Derecho que no incluya los elementos para cuestionar su esfera discursiva, la que indisociablemente se encuentra relacionada a la dominación masculina (en su caracterización objetiva, neutra, apolítica, desinteresada), resulta ser una práctica que desoye deliberadamente las obligaciones asumidas por el estado argentino para modificar los patrones socioculturales que eternizan la subordinación de la mujer. 


\section{De la falta de cumplimiento de las obligaciones legales que implica una educación sin perspectiva de género}

La Convención para la Eliminación de todas las formas de Discriminación contra la Mujer (en adelante CEDAW) define el significado de la igualdad e indica cómo lograrla. Este instrumento constituye un programa de acción que configura una obligación para el Estado argentino por haber adherido a la misma y constituirse entonces como Estado parte. En consecuencia, la Argentina deberá adoptar todas las medidas apropiadas para asegurar el pleno desarrollo de la mujer, con el objeto de garantizarle el ejercicio y el goce de los derechos humanos y las libertades fundamentales en igualdad de condiciones con el hombre (CEDAW, artículo 3). El artículo décimo de la misma normativa establece que los Estados partes adoptarán todas las medidas apropiadas para eliminar la discriminación contra la mujer, a fin de asegurar la igualdad de derechos con el hombre en el ámbito educativo. Del recorrido de los diferentes incisos que componen la norma, destacamos la importancia de la eliminación de todo concepto estereotipado de los papeles masculinos y femeninos en todos los niveles y en todas las formas de enseñanza y en particular, mediante la modificación de los libros y programas escolares y la adaptación de los métodos en enseñanza (CEDAW, artículo 10, inciso c).

En consonancia, la Convención Interamericana para Prevenir, Sancionar y Erradicar la Violencia contra la mujer (en adelante Belem Do Pará) en su artículo sexto inciso b, establece el derecho de toda mujer a una vida libre de violencia, debiendo ser valorada y educada libre de patrones estereotipados de comportamiento y prácticas sociales y culturales basadas en conceptos de inferioridad o subordinación, estableciendo expresamente que los Estados partes convienen en adoptar, en forma progresiva, medidas específicas -inclusive programas- para modificar los patrones socioculturales de conducta de hombres y mujeres, incluyendo el diseño de programas de educación formales y no formales apropiados a todo nivel del proceso educativo, para contrarrestar prejuicios y costumbres y todo otro tipo de prácticas que se basen en la premisa de la inferioridad o superioridad de cualquiera de los géneros o en los papeles estereotipados para los sexos que legitiman o exacerban la violencia contra la mujer.

En el plano nacional, la Ley de Protección Integral a las Mujeres Nro. 26485, sancionada en marzo de 2009, garantiza en su artículo tercero todos los derechos reconocidos por la CEDAW, la convención Belem Do Pará, Convención sobre los Derechos del Niño y la Ley nacional Nro. 20061 de protección integral de los Derechos de las Niñas, Niños y adolescentes. Esta ley de orden público tiene por objeto promover y garantizar, entre otros, la eliminación de la discriminación entre hombres y mujeres, el derecho de las últimas a vivir una vida sin violencia, 
las condiciones aptas para sensibilizar y prevenir, sancionar y erradicar la discriminación y la violencia contra las mujeres en cualquiera de sus manifestaciones y ámbitos, el desarrollo de políticas públicas de carácter interinstitucional sobre violencia contra las mujeres y la remoción de patrones socioculturales que promueven y sostienen la desigualdad de género y las relaciones de poder sobre las mujeres.

La normativa internacional y la legislación interna a la que hemos hecho referencia, en conjunción con los aportes realizados por los feminismos jurídicos, específicamente en relación con la ausencia de una perspectiva de género en la formación de las abogadas y los abogados, ponen de manifiesto una emergencia pedagógica que demanda la reforma de los planes de estudio de las diferentes universidades que dictan la carrera de Abogacía, tal como que venimos remarcando.

Creemos que la toma de decisión política de un Estado obligado por las normas aludidas y que, aun así, decide no brindar herramientas teóricas y metodológicas relativas al género a quienes serán las y los futuros profesionales del Derecho, genera una práctica que contraría abiertamente el derecho positivo, máxime cuando el artículo segundo de la Ley Nro. 24521 de Educación Superior establece la responsabilidad principal e indelegable del Estado nacional, las provincias y la Ciudad Autónoma de Buenos Aires de promover políticas de inclusión educativa que reconozcan igualitariamente las diferentes identidades de género y de los procesos multiculturales e interculturales. Nada de ello sucede en la realidad de las aulas en donde se enseña y se aprende todo lo relativo al mundo jurídico, situación que asegura la reproducción de las desigualdades sociales perpetuando la violencia diaria que ejerce el patriarcado.

En consecuencia, la omisión en los planes de estudio de una perspectiva de género que atraviese el modo de pensar que la universidad enseña -o debería enseñar-, configura un modo de violencia simbólica, a la luz de lo normado por el artículo cuarto de la Ley 26485, toda vez que aquello incide de manera directa e indirecta, tanto en el ámbito público como privado (el ejercicio de la profesión por un lado, la formación de ciudadanos por el otro) en la reproducción de patrones socioculturales que se traducen en la afectación de los derechos humanos afectados por la cuestión de género.

\section{El patriarcado como "ideología sexual"}


El patriarcado como ideología sexual propia de una historia que legitima la subordinación femenina en función de una visión naturalista y esencialista se constituye como un sistema de referencias que explica las relaciones entre hombres y mujeres tomando a uno de los sexos (el masculino) como parámetro de lo humano (Facio y Fries, 2005: 261).

Siguiendo a Bourdieu (2000: 8) conviene recordar que aquello que aparece como eterno en la historia sólo es producto de un trabajo de eternización que incumbe a determinadas instituciones. De allí la necesidad de quebrantar los principios que se muestran como neutrales en el imaginario del estudiante de Derecho desde su formación de grado, para contribuir a cuestionar aquellas instituciones reproductoras de una subordinación que deshistoriza las estructuras de la división sexual y los principios a los que se encuentran ligadas.

Por otra parte, la perspectiva feminista que intentamos incorporar a las Teorías críticas del Derecho, cobra relevancia en su aspecto metodológico, en tanto propone un análisis socio histórico de los mecanismos culturales de dominación y poder cuyos efectos no sólo se extienden a la cuestión de género sino a todo aquello que niegue la alteridad y constituya un "otro". Enfrentarnos al sentido patriarcal de la vida permite evitar que los sexismos se sigan reproduciendo y combustionando diversas formas de negar al otro en su particularidad, como los neofascismos y la fobia a cualquiera que no se identifique con el sector dominante (Facio y Fries, 2005: 261).

\section{Sexo y género: no se nace feminista}

Mientras que el sexo se define por la asignación genital biológica, el género como categoría conceptual constituye

El conjunto de ideas, representaciones, prácticas y prescripciones sociales que una cultura desarrolla desde la diferencia anatómica entre los sexos, para simbolizar y construir socialmente lo que es "propio" de los hombres (lo masculino) y lo que es "propio" de las mujeres (lo femenino) (Lamas, 2000: 2).

De esto modo la categoría de género se constituye como un modo de dar cuenta de las lógicas relacionales, culturales y políticas que se establecen a partir de la diferencia sexual (Costa, 2016: 69). 
En El segundo sexo, Simone De Beauvoir (2007) afirma que "no se nace mujer, se llega a serlo" buscando evidenciar la incidencia de la cultura en la conformación de las diferencias entre hombres y mujeres, dando cuenta a su vez de la condición subalterna de lo femenino.

Este posicionamiento de las mujeres en los márgenes de una sociedad patriarcal es aprendido por los sujetos a través de los procesos primarios y secundarios de socialización, que dan lugar a la asimilación de la dominación. En el caso de las mujeres, aquel conocimiento se transforma en reconocimiento práctico del código que transmite la lógica de los dominadores a través de la violencia simbólica, siendo la universidad en este caso una pieza fundamental del sistema de reproducción de las desigualdades de género.

Entender que el sexo y el género son categorías independientes, una proveniente del universo de lo biológico y otra de lo cultural -las que a su vez pueden coincidir o no- constituye el eslabón fundamental para el respeto de la diversidad de las experiencias humanas y el cuestionamiento del rol subalterno de las mujeres basado en la diferencia sexual.

Teniendo en cuenta las incumbencias profesionales y sociales de las abogadas y los abogados como constructores y manipuladores de la ley, una enseñanza en clave de género se orienta a develar el poder paradojal del discurso jurídico, aquel que denuncia la versatilidad en tanto puede constituirse como un reproductor de las estructuras que habilitan la dominación masculina pero también, puede en un instrumento de transformación social.

Es por ello que formar a las abogadas y a los abogados en perspectiva de género implica, ni más ni menos que respetar el trinomio de indivisibilidad, interdependencia y progresividad que reconocen los Derechos Humanos cuyo único requerimiento para su titularidad es el hecho de ser persona (Costa, 2016: 119).

\section{Género y Derecho. ¿Qué hacen los abogados?}

Como ya dijimos, entendemos al Derecho como un discurso prescriptivo y paradójico, con la capacidad de reconocer e invisibilizar, incluir y excluir las categorías de lo legal y lo ilegal (Costa, 2016: 11). Los feminismos jurídicos apuntan a desnaturalizar el discurso jurídico y a cuestionar la "neutralidad ideológica" que sostienen las corrientes conservadoras de corte (neo)liberal, apuntando en el caso de los feminismos jurídicos a la necesidad insoslayable de entender al Derecho como praxis, como teoría indisociable de la acción.

Comprender el significado de los procesos de socialización que ocurren en las aulas de las facultades de Derecho adquiere singular relevancia, dadas las particularidades de las 
incumbencias propias de las abogadas y los abogados. En resumidas cuentas, estas y estos profesionales poseen a su cargo la construcción y el mantenimiento del orden social (cuyo fundamento reposa en el discurso jurídico) por lo que se constituyen como un grupo de poder para ejercer e influir en el mundo de la cultura (juezas y jueces, fiscales, defensoras y defensores públicos y particulares, abogadas y abogados que ejercen la profesión autónomamente) destacando a su vez la omnipresencia de una profesión que se encuentra presente en todos los ámbitos de la sociedad (Brígido, 2014: 14).

Dadas estas particularidades y la influencia de estas y estos profesionales en los mecanismos de reproducción social, insistimos en la emergencia que atañe a la formación universitaria de los mismos. El cuestionamiento del discurso pedagógico resulta clave para la redefinición de las condiciones de ejercicio de las prácticas de reproducción del discurso jurídico dentro del sistema educativo (Bernstein y Díaz, 2017).

\section{Reflexiones finales}

El presente trabajo busca constituirse como una aproximación preliminar a los interrogantes que puedan suscitarse a partir de las reflexiones sobre la crítica feminista de la representación de una humanidad universal para transformar los modos de conocimiento establecidos (Costa, 2016: 13) a la vez que pretende remarcar la pertinencia espacial de la universidad para ese propósito. Remarcamos así la necesidad de una pedagogía atenta al diálogo entre la construcción social de la realidad y el discurso jurídico como expresión de poder, para pensar una enseñanza del Derecho en clave feminista con capacidad para hacer frente a las resistencias del mundo académico/jurídico frente a este universo teórico que lucha por la igualdad de las personas, desafiando las fronteras de los géneros y velando por el efectivo respeto de los Derechos Humanos.

Señalamos que la postura crítica del Derecho que asumen los feminismos jurídicos toma en cuenta el carácter socio histórico del mismo como discurso que (re)produce las condiciones materiales de la existencia, perpetuando privilegios y negando las consecuencias de las diferencias socialmente construidas en torno a las trayectorias de hombres y mujeres en su especificidad de género. Es por ello que consideramos oportuno poner el foco en la singularidad paradójica del discurso jurídico, ya que, si bien legitima las relaciones de poder existentes, sirve asimismo para su transformación. Es nuestra tarea entonces la de emprender 
una batalla ideológica para poder vivir en una real democracia que respete los derechos que proclaman sus textos normativos.

Por último, esperamos que las reflexiones compartidas puedan abrir el incipiente debate en torno a la pregunta que formula McKinnon (2005): “ ¿...qué puede hacer la enseñanza del Derecho para formar abogados que intervengan en esta situación -la desigualdad de las mujeres respecto de los varones- y así transformarla?"

\section{Notas}

(1) Son aportes útiles para pensar el carácter conservador de las facultades de Derecho, los señalamientos realizados por Andriola y Goga (2014).

(2) La denominación de esta área es diversa: Feminist Legal Theory, Feminist Legal Thought, Feminist Jurisprudence. Para una cronología del tema, véase Costa, 2016.

\section{Bibliografía}

Andriola, K. y Goga, D. (2014). Las mujeres en la Facultad de Ciencias Jurídicas y Sociales. En González, M. y Marano, M. G. (comp). La formación de abogadas y abogados. Nuevas configuraciones.

http://www.icj.jursoc.unlp.edu.ar/documentos/la_formacion_de_abogados.pdf

Berger, P. y Luckmann T. (2003). La construcción social de la Realidad. Buenos Aires: Amorrotu Editores.

Bernstein, B. y Díaz, M. (2017). Hacia una teoría del discurso pedagógico. Revista colombiana de educación, $15 . \quad$ Recuperado de http://revistas.pedagogica.edu.co/index.php/RCE/article/view/5120/4199

Bourdieu, P. (2000). La dominación masculina. España: Editorial Popular.

Brígido, A. M. (2014). Prólogo. En González, M. y Marano, M. G. (comp.). La formación de abogadas y abogados. Nuevas configuraciones. Recuperado de http://www.icj.jursoc.unlp.edu.ar/documentos/la_formacion_de_abogados.pdf

Convención Interamericana para Prevenir, Sancionar y Erradicar la Violencia contra la Mujer "Convención De Belem Do Para". Departamento de Derecho Internacional, OEA. Recuperado de http://www.oas.org/juridico/spanish/tratados/a-61.html 
Convención para la Eliminación de todas las formas de Discriminación contra la Mujer (CEDAW). http://www.un.org/womenwatch/daw/cedaw/text/sconvention.htm

Costa, M. (2016). Feminismos jurídicos. Buenos Aires: Ediciones Didot.

De Beauvoir, S. (2007). El segundo sexo. Buenos Aires: Sudamericana.

Facio, A. y Fries, L. (2005). Feminismo, género y patriarcado. Academia. Revista sobre enseñanza del Derecho en Buenos Aires, 3(6), pp. 259-294.

Foucault, M. (1981). The order of discourse. En Young, E. (ed.). Untying the Text: A PostStructuralist Reader. London: Routledge and Kegan Paul.

Lamas, M. (2000). Diferencias de sexo, género y diferencia sexual. Cuicuilco, 7(18), Escuela Nacional de Antropología e Historia Distrito Federal, México.

Ley 20061. Ley de protección integral de los derechos de las Niñas, Niños y Adolescentes, 21 de octubre de $2005 . \quad$ Recuperado de http://servicios.infoleg.gob.ar/infolegInternet/anexos/110000-114999/110778/norma.htm

Ley 24521. Ley de Educación Superior, 7 de agosto de 1995. Recuperado de http://servicios.infoleg.gob.ar/infolegInternet/anexos/25000-29999/25394/texact.htm

Ley 26485. Ley de protección integral a las mujeres, 1 de abril de 2009. Recuperado de http://servicios.infoleg.gob.ar/infoleglnternet/anexos/150000-154999/152155/norma.htm

Maffia, D. (2012). Hacia un lenguaje inclusivo ¿Es posible? Jornadas de actualización profesional sobre traducción, análisis del discurso, género y lenguaje inclusivo. Recuperado de https://bit.ly/2JdnXQr

McKinnon, K. (2005). Integrando el feminismo en la educación jurídica. Academia. Revista sobre enseñanza del Derecho, 3(6) pp.157-174. Recuperado de https://bit.ly/2uGBVqv 